\section{Commentary: Surgery for rheumatic heart disease in Africa: Excellence is attainable, but questions remain}

\author{
R. Morton Bolman III, MD
}

The manuscript by Tamirat and colleagues ${ }^{1}$ describes a nearly 10-year experience with surgery for rheumatic heart disease (RHD) in Ethiopia, examining 240 cases operated by 24 surgeons during 22 humanitarian missions carried out between 2009 and 2017. All patients had disease involving the mitral or the aortic valve or both. The goal of the study was to compare outcomes for 3 different operative strategies: valve repair or valve replacement, and if the latter, with a mechanical versus a biologic prosthesis. The primary outcome was all-cause mortality, and the secondary outcome was major adverse valve-related event (MAVREs), a composite of death, reoperation, stroke, major bleeding, endocarditis, and structural valve deterioration (SVD).

With a median patient age of 19.8 years ( $57 \%$ female), $90(38 \%), 58(24 \%)$, and $92(38 \%)$ patients, respectively, received mechanical replacement, bioprosthetic replacement, or valve repair. There were 5 reoperations.

Operative mortality occurred in 5 patients $(2.1 \%)$, and there was no significant difference among the procedure groups. An additional 11 patients died in follow-up. Fifty-five patients (23\%) experienced at least 1 MAVRE.

At a median follow-up of 2.3 years, a greater mortality was observed in the patients receiving bioprosthetic valves. There was no difference in mortality between patients receiving mechanical prostheses and those undergoing repair. Patients with bioprosthetic valves also experienced significantly more MAVREs, whereas the rates of MAVRE

\footnotetext{
From the Division of Cardio-Thoracic Surgery, Department of Surgery, University of Colorado, Aurora, Colo.

Disclosures: The author reported no conflicts of interest.

The Journal policy requires editors and reviewers to disclose conflicts of interest and to decline handling or reviewing manuscripts for which they may have a conflict of interest. The editors and reviewers of this article have no conflicts of interest.

Received for publication April 1, 2020; revisions received April 1, 2020; accepted for publication April 1, 2020; available ahead of print April 18, 2020.

Address for reprints: R. Morton Bolman III, MD, 7070 W Belmont Dr, Littleton, CO 80123 (E-mail: cbolman46@gmail.com).

J Thorac Cardiovasc Surg 2021;162:1726-7

$0022-5223 / \$ 36.00$

Copyright $(2) 2020$ by The American Association for Thoracic Surgery

https://doi.org/10.1016/j.jtcvs.2020.04.021
}

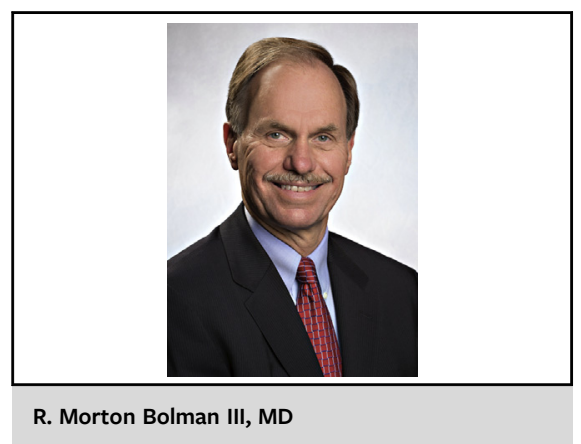

CENTRAL MESSAGE

A report of surgery for $\mathrm{RHD}$ in Ethiopia explored the advantages and disadvantages of various operative strategies over a 2.3-year follow-up. Lessons are derived that can inform future studies.

were similar in patients receiving mechanical valves and those undergoing repair. Four-year freedom from MAVREs was $87 \%, 55 \%$, and $56 \%$, respectively, for patients receiving mechanical replacement, repair, and bioprosthetic replacement. Notably, at a follow-up of only 2.3 years, $16 \%$ of bioprosthetic valves had experienced SVD.

This paper demonstrates a number of the realities and challenges facing surgery for RHD in sub-Saharan Africa. First, it is feasible for experienced practitioners to perform complex open heart surgery, even in these austere settings, with acceptable mortality and morbidity rates. Nearly $50 \%$ of the operations in this report were double- or triple-valve procedures. These can prove challenging, even in developed countries. The operative outcomes were excellent.

There are a number of factors specific to this experience that make it difficult to extrapolate the outcomes to other countries or situations. These include, in no particular order or ranking, the fact that these 240 operations were performed by 24 different surgeons, yielding an average of just 10 operations per surgeon. There was a variable interest/expertise in valve repair among the surgeons involved, expressed as "some of the surgeons were very committed to valve repair." When valve replacement was selected, the choice of prosthesis was based on 3 factors: patient factors, including desire for childbirth, socioeconomic factors influencing the ability to comply with an anticoagulation regimen, and, last but not least, limited availability of valves in the humanitarian setting. 
I respectfully contend that, with this number of surgeons involved, with variable interest and expertise in valve repair, with prosthesis choice not protocol- or policy-driven, and partly determined by mere availability, not much further can be concluded from this experience. As the authors correctly point out, bioprosthetic valves are disadvantageous for this population of patients and are likely to fail within 2 to 5 years at an unacceptable rate.

The authors also correctly observe that reoperations are rare in this setting and will likely remain so for the foreseeable future. For that reason, as well as the $29 \%$ incidence of SVD observed in the patients undergoing repair in this series, at a follow-up of only 2.3 years, one must be cautious in equating the outcomes of mechanical replacement, with $0 \% \mathrm{SVD}$ in this series, with valve repair. This despite the greater rate of stroke and major bleeding observed in patients receiving mechanical valves.

There are some lessons that have emerged from the current, as well as other, reported experiences with surgery for RHD in Africa. First, in this young population, bioprosthetic valves are suboptimal as replacement devices, because of a propensity to fail at an accelerated rate. Second, mechanical valves offer a lasting solution to the pathology encountered in RHD, albeit at the price of lifelong anticoagulation. As the authors correctly observe, management of anticoagulation in resource-poor settings is a significant barrier, and health care systems in countries offering these procedures must be challenged to raise the level of care to meet the needs of these patients. Last, but not least, valve repair may one day offer the best solution to this conundrum. This will only be true, however, if and when techniques can be developed and taught consistently to the surgeons on the frontlines in low- and middleincome countries, which reliably and durably correct the valvular pathology that will be encountered in areas of endemic RHD. Certainly, this is not the case today, in 2020. Second and subsequent operations for failed valve repairs will not be routinely available in settings such as exemplified in the current series for many years to come.

Finally, I would comment that although the authors commented that the Cardiac Center of Ethiopia has on its staff 2 cardiac surgeons, there is no mention of how these individuals participated in the surgeries that are herein reported. In the spirit of the Cape Town Declaration, ${ }^{2}$ which dedicates the resources of the cardiac surgery, cardiology, medical device industry, and the governmental communities to increasing access to cardiac surgery and providing training to critical providers in low- and middle-income countries, one would certainly hope and anticipate that every effort would have been made to mentor these surgeons with the goal of establishing sustainable cardiac surgery in Ethiopia, eventually without the need of visiting humanitarian teams. All of us in this field look forward to the day when the local teams will be able to successfully operate independently, and the services of expatriate, visiting humanitarian teams will no longer be required.

\section{References}

1. Tamirat S, Mazine A, Stevens L-M, Agwar F, Dejene K, Bedru M, et al. Contemporary outcomes of aortic and mitral valve surgery for rheumatic heart disease in sub-Saharan Africa. J Thorac Cardiovasc Surg. 2021;162:1714-25.e2.

2. Zilla P, Bolman RM, Yacoub M, Beyersdorf F, Sliwa K, Zuhlke L, et al. The Cape Town declaration on access to cardiac surgery in the developing world. J Thorac Cardiovasc Surg. 2018;156:2206-9. 